\section{TONSIL SNARE.}

\section{EDWARD C. SEWALK, M.D.}

SAN FRANCISCO.

The use of the snare with a cold steel wire for the removal of the tonsil requires an instrument simple in construction and strong in all parts. The instrument here shown covers, I think, these requisites.

No provision is made for the re-use of the same wire because the snare can be so quickly threaded and a loop once used loses its best form. The general shape and mechanism is that of snares that are well known to be satisfactory. The parts have been adapted to the uses to which it is to be put. The canula is strong and has a button on the end to prevent its splitting. The screw for the attachment of the wire is set firmly into a strong

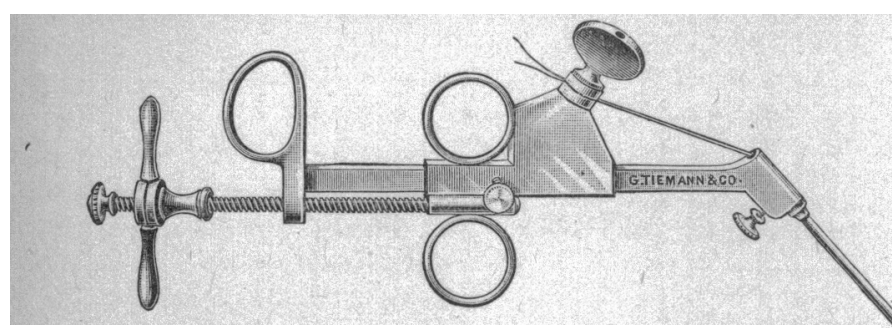

piece of steel; over this fits a button and then a loose washer. Both button and washer are made of chilled steel and milled so that by means of the strong thumbscrew the wire can be secured and quickly fastened. Most snares fail to provide for the quick and secure fastening of the wire, it often being made fast by clumsy and slow twisting. When the loop has engaged the tonsil the wire is drawn taut and then the cutting slowly accomplished by the windlass. This crushes the vessels and makes bleeding less to be feared.

\section{CHRYSOPHANIC ACID KERATO-CONJUNC- TIVITIS.}

\section{CHARLES J. KIPP, M.D.} NEWARK, N. J.

The fact that chrysophanic acid or chrysarobin, when applied in the form of a salve to any part of the face and even to other parts of the body, will give rise in some individuals to a conjunctivitis of greater or less severity is known to dermatologists and is mentioned in many text-books on diseases of the skin. Recently this drug seems to have come into favor again, at least I have been told by apothecaries that it is prescribed more frequently now than for several years past, and this is one reason which has induced me to put the following case on record:

Patient.-N. C. L., aged 63, in good health, of temperate habits, a clerk by occupation, came to me on May 5, 1906.

Examination.-He was led into my office with a bandage over his eyes, and his companion told me that he was blind. I found that the eyelids of both eyes were red, shining and edematous. The edges of the lids were glued together by secretions. After soaking the lashes with warm water, the lids were opened and tears streamed from the conjunctival sac. There was not much secretion in the conjunctival sac. The palpebral conjunctiva was very red and swollen, the bulbar conjunctiva was edematous and intensely injected. The cornex of both eyes were hazy, but the epithelial layer was apparently intact. The pupil was small, but there was no iritis and no disease of the posterior half of the eye. Both eyes were in about the same condition. The patient was complaining bitterly of pain and intense burning in his eyes and of photo- phobia. He was unable to keep his eyes open long enough to determine the refraction of the eyes and the amount of vision.

History. - This is the first time in his life that his eyes have been inflamed. For some years the tip of his nose has been somewhat red from enlargement of the blood vessels in the skin, and this has annoyed him a good deal, especially as he never indulges in alcoholic drinks. He was told that this condition could be removed, and thereon he consulted his family physician, a homeopath, who prescribed a salve which was to be applied to the skin of the nose every night and washed away again the following morning. He applied this salve only once, and on the following morning tried to wash it off, and in doing so he probably got some of it in his eyes. His eyes, which previous to this time had been in normal condition, began to give him pain soon afterward, and the lids began to swell in the afternoon of the same day. The pain and the swelling of the lids increased very much during the following night. He slept but little in consequence of the pain. I saw him fortyeight hours after he had rubbed the salve in his eyes. He showed me the salve he had been using. It looked like chrysarobin salve, but to make sure of its composition I applied to the apothecary who had compounded the salve for a copy of this prescription. It showed the salve to be composed of chrysophanic acid $3 \mathrm{ii}$ and lard $3 \mathrm{vi}$.

Treatment.-An examination of the secretion from the conjunctiva showed it to contain a few pneumococci. I advised the patient to apply cold compresses to his lids, a salve of am. moniated mercury to the edges of the lids, and to put a drop of a 0.5 per cent solution of sulphate of atropin and 1 per cent. cocain in each eye six times daily. The cold application failed to give relief, and I substituted for them compresses wet with a warm solution of boracic acid. During the changed. I brushed the excoriated edges of the lids with a I per cent. solution of nitrate of silver once daily. On the third day the epithelium of the cornea was wanting in a number of places, mostly in the horizontal meridian, and in the right eye somewhat more than in the left, and the whole cornea appeared somewhat more hazy. The lids were now less swollen and much paler than previously. On the fifth day the deficiency in the epithelial layer of the cornea extended across the whole cornea and was about $1 \mathrm{~mm}$. in width. The adjoining parts of the cornea were decidedly hazy; both eyes were in about the same condition. At this time there was but little secretion from the conjunctiva. On the sixth day the entire lower half of the cornea of the right eye was very hazy. The deficiency of the epithelial layer had not increased in extent. The left eye remained as before. He had but little pain, but the photophobia continued. On the ninth day there was a decided improvement in the condition of the cormex of both eyes. There was less haziness, and the deficiency in the epithelial layer was gradually filling in. On the fourteenth day the corneæ were clear and there was no longer a defect in the epithelial layer, the lids were normal or very nearly so, and there was no secretion from the conjunctiva. He was free from pain, but still had photophobia. On the twentieth day both eyes were normal. The refraction was hypermetropia 2 D. S. 6/5, both eyes alike.

There can be no doubt, I think, that the eye disease was caused by the entrance of the chrysarobin salve in the conjunctival sac. Whether the corneal disease was due to the direct action of the chrysarobin or was caused by the conjunctivitis, I am unable to say. The disease of the cornea certainly made the case a much more serious one, as it endangered the sight of the eye. Fortunately, it passed away without leaving even a slight opacity of the cornea.

With regard to the treatment given in this case, I may add that $I$ should not use cocain in such a case unless the pain could not be relieved by the other means employed. The tendency to exfoliation of the epithelium of the cornea is always great in such cases and is 